\title{
Sensitivity of the ANTARES neutrino telescope to atmospheric neutrino oscillation parameters
}

\author{
I. Salvadori* \\ Aix Marseille Univ, CNRS/IN2P3, CPPM, Marseille, France \\ E-mail: salvadoriecppm.in2p3.fr
}

\section{On behalf of the ANTARES Collaboration}

\begin{abstract}
A new analysis, aimed at improving the current ANTARES measurement of the atmospheric neutrino oscillation parameters, is presented in these proceedings. Two different track reconstruction procedures are combined in order to increase the sensitivity. Furthermore, a novel method to estimate the neutrino energy is applied. A complete 3-flavour description of the oscillation probability including matter effects in the Earth is used. By performing a two-dimensional fit of the event rate as a function of reconstructed energy and zenith angle, expectations on the sensitivity to the oscillation parameters are derived. Using the same analysis chain, a study on the ANTARES sensitivity to sterile neutrinos is performed.
\end{abstract}

35th International Cosmic Ray Conference - ICRC2017-

10-20 July, 2017

Bexco, Busan, Korea

\footnotetext{
*Speaker.
} 


\section{Introduction}

The ANTARES neutrino telescope [1] is situated in the Mediterranean Sea, $40 \mathrm{~km}$ from the coast of Toulon (France). It is composed of 12 detection lines, each one equipped with 25 floors of 3 optical modules (OMs), with a vertical spacing of $14.5 \mathrm{~m}$. The horizontal spacing among the lines is around $60 \mathrm{~m}$. The main goal of ANTARES is the observation of high energy neutrinos from galactic and extra-galactic sources. The detector is, for this reason, optimized to detect neutrinos of energies up to $\mathrm{TeV}$, by detecting the Cherenkov photons emitted by charged particles produced in neutrino interactions. On the other hand, at neutrino energies of the order of $\mathrm{GeV}$, the detector configuration and the reconstruction algorithms allow to study the phenomenon of atmospheric $v_{\mu}$ disappearance due to neutrino oscillations, and constraints on atmospheric neutrino oscillation parameters can be derived.

The document is organized as follows: in Section 2 the mechanism of neutrino oscillations is recalled; in Section 3 the MC simulations are described; the track and energy reconstruction procedures are explained in Section 4, together with the details of the event selection; a discussion of the minimization procedure is described in Section 5 and the ANTARES sensitivity for the $\Delta m_{32}^{2}$ and $\theta_{23}$ parameters are presented in Section 6. In Section 7 the parameter space which can be tested under the assumption of the existence of a sterile neutrino is obtained. Conclusions and prospects are given in Section 8.

\section{Neutrino Oscillations}

Neutrino oscillation is a quantum mechanical phenomenon, which occurs since the neutrino mass eigenstates $\left(v_{1}, v_{2}, v_{3}\right)$, which are used to describe neutrino propagation through space, are not the same as the neutrino flavour eigenstates $\left(v_{e}, v_{\mu}, v_{\tau}\right)$, which are the ones taking part in interactions. The relation between these two bases is described by the Pontecorvo-Maki-Nakagawa-Sakati (PMNS) matrix [2], and can be parameterized by three mixing angles $\left(\theta_{12}, \theta_{23}, \theta_{13}\right)$ and a CP violating phase $\left(\delta_{C P}\right)$.

The survival probability of atmospheric $v_{\mu}$ in the 3 flavour scenario can be written as:

$$
\begin{aligned}
P_{v_{\mu} \rightarrow v_{\mu}}=1 & -4\left|U_{\mu 1}\right|^{2}\left|U_{\mu 2}\right|^{2} \sin ^{2}\left(\frac{1.27 \Delta m_{12}^{2} L}{E}\right) \\
& -4\left|U_{\mu 1}\right|^{2}\left|U_{\mu 3}\right|^{2} \sin ^{2}\left(\frac{1.27 \Delta m_{31}^{2} L}{E}\right) \\
& -4\left|U_{\mu 2}\right|^{2}\left|U_{\mu 3}\right|^{2} \sin ^{2}\left(\frac{1.27 \Delta m_{32}^{2} L}{E}\right)
\end{aligned}
$$

where $L$ is the distance traveled by the neutrino (in $\mathrm{km}$ ), $E$ is its energy (in $\mathrm{GeV}$ ), $U_{\alpha i}$ are the elements of the PMNS matrix, and $\Delta m_{i j}^{2}=\left|m_{i}^{2}-m_{j}^{2}\right|\left(\right.$ in $\left.\mathrm{eV}^{2}\right)$ is the absolute difference of the squares of the mass eigenstates.

In this work, a complete 3 flavour framework is used, and the survival probabilities of atmospheric muon neutrinos including Earth matter effects are computed numerically using the open source software OscProb [3].

\section{Monte Carlo Simulations}

The official Monte Carlo (MC) production of ANTARES has been used for this work, which simulates individual data acquisition "runs" from 2007 to 2015. A total lifetime of around 2236 
days has been considered. Neutrino events are generated using the GENHEN [4] software, developed in the ANTARES Collaboration, and they are weighted with the Honda atmospheric neutrino flux [5], if not otherwise specified. For the simulation of atmospheric muon bundles, instead, the MUPAGE [6] software is used. Atmospheric muons wrongly reconstructed as up-going represent the main background source for this study. This program uses parametric formulas to describe the energy, zenith and multiplicity distributions of muons arriving at the detector [7].

\section{Event Reconstruction and Selection}

Two different track reconstruction algorithms have been combined in the analysis, in order to increase the sensitivity. A detailed description of the procedures can be found in [9] and [10], respectively.

Starting from the reconstructed muon direction, a hit selection based on spatial and time distributions is made, in order to select direct Cherenkov photons coming from the muon track. The photons are then projected back to the track to find the first and last emission point, which are then used for the muon track length estimation. Taking into account the ionisation energy loss of 0.24 $\mathrm{GeV} / \mathrm{m}$ for minimum ionising muons in sea water, the muon energy is computed.

In order to reduce the contamination due to atmospheric muons, only events reconstructed as upward-going are considered in the analysis. Requiring a containment condition, based on the position of the reconstructed interaction vertex, and applying additional cuts based on quality parameters specific for each reconstruction procedure, the remaining background decreases to about 0.03 muons per day. Additional sources of background are due to neutral-current events, but these are negligible. The atmospheric neutrino signal is expected to be of around 3 neutrinos per day.

\section{Minimization Procedure}

MC events have been binned in a 2-dimensional histogram with 100 logarithmic bins in reconstructed muon energy, from 0.1 to $200 \mathrm{GeV}$, and 21 bins in reconstructed cosine of the zenith angle, from -1 to 0.2 . A pseudo-data sample, i.e. a number of events with the same characteristic of a real data sample collected in the detector lifetime of 2236 days, has been created using MC simulations assuming the values of atmospheric oscillation parameters $\Delta m_{32}^{2}=2.43 \times 10^{-3} \mathrm{eV}^{2}$ and $\theta_{23}=41.5^{\circ}$. We chose such a value of $\theta_{23}$ because is close to the current global best fit value for this parameter [11]. The expected sensitivity of the analysis to the oscillation parameters has then been tested by computing the $\chi^{2}$ for each different hypothesis with respect to the test point.

The Earth density profile has been paramterized using the PREM model [12]. The ROOT class Minuit2Minimizer has been used to perform the $\chi^{2}$ minimization in order to find the values of the atmospheric neutrino oscillation parameters which fit best our pseudo-data sample. A normal neutrino mass ordering is assumed.

The minimization procedure depends on multiple parameters which affect the oscillation pattern in zenith and energy, apart from the oscillation parameters themselves. So far, only a global normalization factor, $N$, is left free to account for these systematic effects. Also $\theta_{13}$ has been fitted, but with a prior. In Table 1 a complete list of all the fitted parameters together with their test values and eventual prior is presented. 


\begin{tabular}{ccc}
\hline Parameter & Test Point Value & Prior \\
\hline $\mathrm{N}$ & 1.00 & FREE \\
$\Delta m_{32}^{2}\left[10^{-3} \mathrm{eV}^{2}\right]$ & 2.43 & FREE \\
$\theta_{23}\left[{ }^{\circ}\right]$ & 41.50 & FREE \\
$\theta_{13}\left[{ }^{\circ}\right]$ & 8.41 & $8.41 \pm 0.28$ \\
$\theta_{12}\left[{ }^{\circ}\right]$ & 33.46 & FIXED \\
$\Delta m_{21}^{2}\left[10^{-5} \mathrm{eV}^{2}\right]$ & 7.53 & FIXED \\
$\delta_{C P}\left[{ }^{\circ}\right]$ & 0.00 & FIXED \\
\hline
\end{tabular}

Table 1: Oscillation parameters (first column), values used to construct the pseudo-data sample (second column) and eventual prior (third column).

\section{ANTARES Sensitivity to the Atmospheric Neutrino Oscillation Parameters}

The sensitivity region is presented in form of confidence intervals in the parameter space $\theta_{23}-$ $\Delta m_{32}^{2}$, which have been computed by looping on a fine grid of values around the minimum, and using tabulated critical values for $\chi^{2}$ with 2 degrees of freedom (dof). In Figure 1 the sensitivity at $90 \%$ C.L. obtained from the analysis of the pseudo-data sample used is compared with the results from other experiments. The one-dimensional contours are also shown. They have been obtained by looping over the same grid of values of $\Delta m_{32}^{2}$ and $\theta_{23}$, respectively, and minimizing with respect to the other free parameters of the fit.

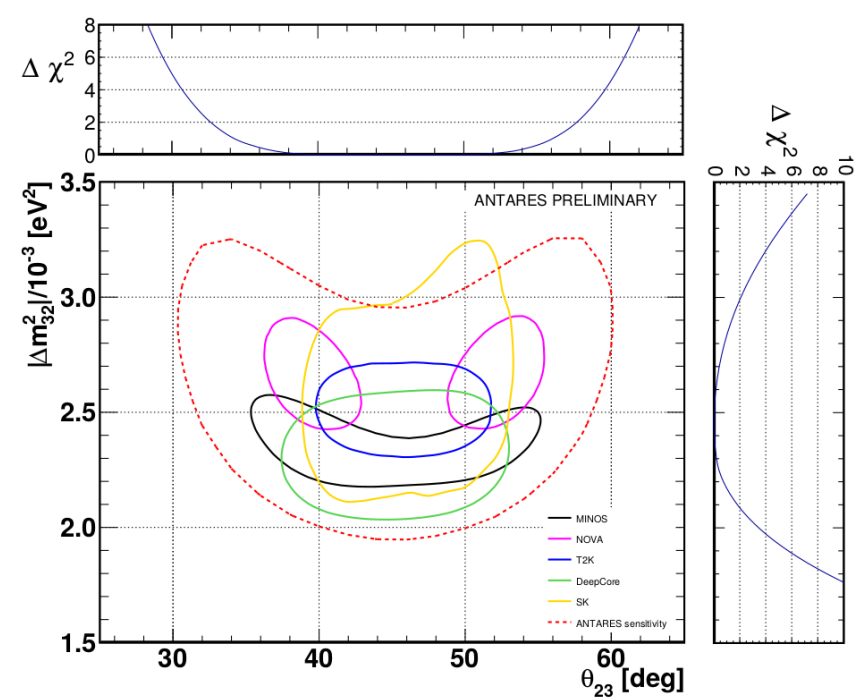

Figure 1: Result obtained from our pseudo-experiment simulating 2236 days of ANTARES lifetime. The red-dashed curve represents the allowed parameter region at $90 \%$ C.L. The one-dimensional contours for the two oscillation parameters under study are also shown. For comparison, results from MINOS [13], NOvA [14], T2K [15], IceCube (DeepCore) [16] and Super Kamiokande [17] are also shown.

\section{ANTARES Expected Sensitivity to Sterile Neutrinos}

The existence of additional neutrinos, which do not take part in the weak interaction but whose 
presence could introduce distortions in the oscillation probability patterns, has been posited as a possible explanation for some neutrino experiment anomalies [18]. Here, only the simplest $3+1$ model is considered, which describes the mixing between the three active neutrinos with only one sterile neutrino.

Figure 2 illustrates the effect that the existence of such a sterile neutrino would have on the oscillation probability of a vertically up going muon neutrino crossing the Earth, as a function of its energy.
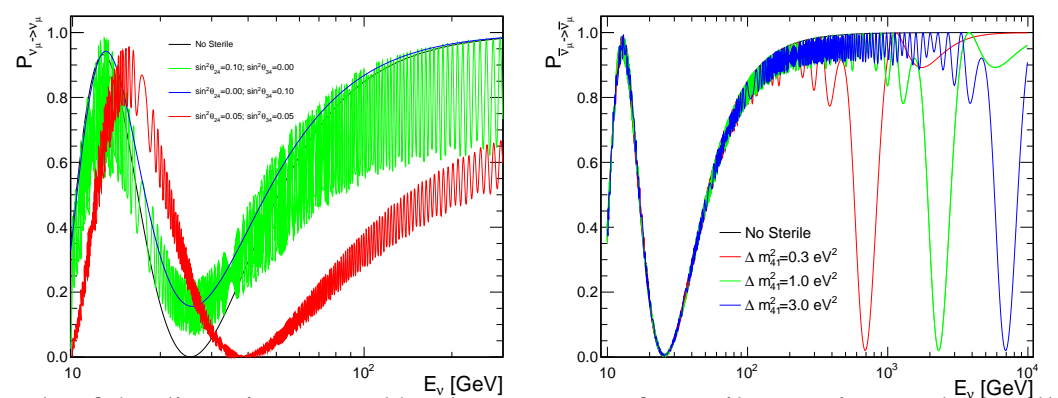

Figure 2: Example of the distortion caused by the presence of a sterile neutrino on the oscillation probability pattern, for a vertical up going $v_{\mu}$ (left panel) and $\bar{v}_{\mu}$ (right panel), as a function of the neutrino energy.

As shown in the figure, two different energy ranges can be studied. The first one, around $20-30 \mathrm{GeV}$ is sensitive to the mixing angles $\theta_{24}$ and $\theta_{34}$, while the second one, around $10^{3} \mathrm{GeV}$, allows to constrain the mass splitting $\Delta m_{41}^{2}$.

Two parallel analyses, described in 7.1 and 7.2 respectively, have been made, in order to study the sensitivity of ANTARES to the sterile neutrino parameters. Data have been imitated with MC assuming no sterile; the standard oscillation parameters have been kept fixed to the same values as illustrated in Table 1, and a global normalization factor, $N$, has been left free to account for systematic effects. The details are presented in the following sub sections.

\subsection{Low Energy Analysis}

For the lowest energy range, the same events from the considered pseudo-data sample as for the standard oscillation analysis are used. Events have been binned in a 2D histogram with 10 logarithmic bins in reconstructed energy, from $10^{0.8} \mathrm{GeV}$ to $10^{2} \mathrm{GeV}$ and 8 bins in reconstructed $\cos \theta$, from -1 to 0 .

In Table 2 a complete list of all the fitted parameters together with their test values and eventual prior is presented.

\begin{tabular}{ccc}
\hline Parameter & Test Point Value & Prior \\
\hline $\mathrm{N}$ & 1.00 & FREE \\
$\theta_{24}\left[{ }^{\circ}\right]$ & 0.00 & FREE \\
$\theta_{34}\left[{ }^{\circ}\right]$ & 0.00 & FREE \\
$\theta_{14}\left[{ }^{\circ}\right]$ & 0.00 & FIXED \\
$\Delta m_{41}^{2}\left[\mathrm{eV}^{2}\right]$ & $\mathrm{N} / \mathrm{A}$ & FIXED at 0.5 \\
\hline
\end{tabular}

Table 2: Oscillation parameters (first column), values used to construct the pseudo-data sample (second column) and eventual prior (third column). 
A $\chi^{2}$ test has been performed and sensitivity confidence intervals on the reduced parameter space $\sin ^{2} \theta_{24}-\sin ^{2} \theta_{34}$ have been built, by looping over a fine grid of values around the minimum, and using tabulated critical values for $\chi^{2}$ with 2 dof. Results are shown in Figure 3.

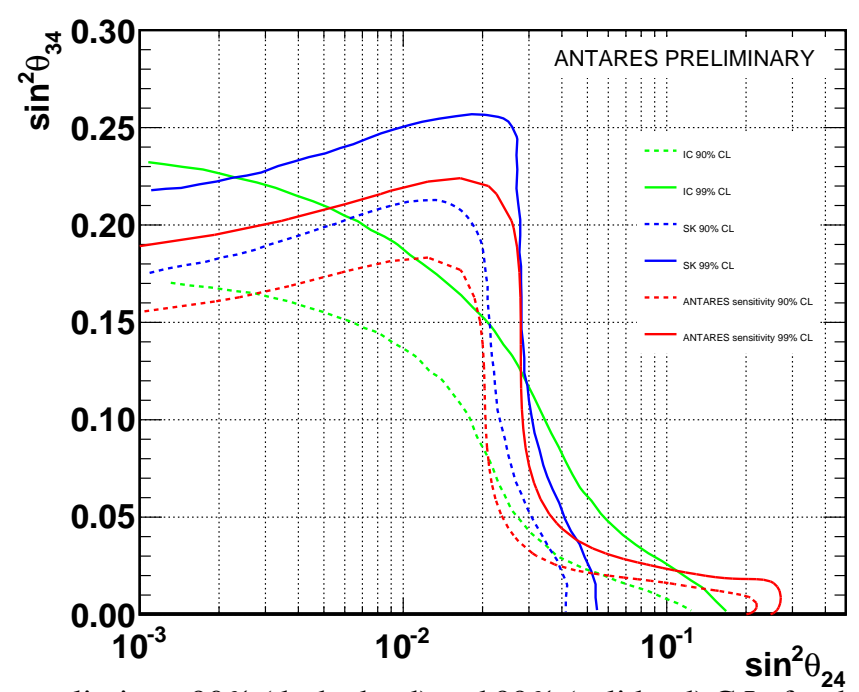

Figure 3: Expected upper limits at 90\% (dashed red) and 99\% (solid red) C.L. for the analysis of our pseudodata sample. Exclusion regions are on the right side of the lines. For comparison limits from IceCube [19], and from Super Kamiokande [20] are also shown.

It is worth to remind that the standard oscillation parameters have been kept fixed for the moment. These parameters are expected to induce the largest uncertainties in this analysis. The next step in this study will be the inclusion of the neutrino mixing parameters, $\Delta m_{32}^{2}$ and $\theta_{23}$, as free parameters in the fit.

\subsection{High Energy Analysis}

For the analysis at higher energies, events reconstructed by a dedicated track reconstruction algorithm, specific for more energetic events, have been considered. The Bartol model for the atmospheric neutrino flux [21] has been used to weight these events. A 2D fit in 10 logarithmic bins from $100 \mathrm{GeV}$ to $10 \mathrm{TeV}$ and 21 bins from -1 to 0.24 in $\cos \theta$ has been performed.

In Table 3 a complete list of all the fitted parameters together with their test values and eventual prior is presented.

\begin{tabular}{ccc}
\hline Parameter & Test Point Value & Prior \\
\hline $\mathrm{N}$ & 1.00 & FREE \\
$\theta_{24}\left[^{\circ}\right]$ & 0.00 & FREE \\
$\theta_{34}\left[{ }^{\circ}\right]$ & 0.00 & FIXED \\
$\theta_{14}\left[{ }^{\circ}\right]$ & 0.00 & FIXED \\
$\Delta m_{41}^{2}\left[\mathrm{eV}^{2}\right]$ & $\mathrm{N} / \mathrm{A}$ & FREE \\
\hline
\end{tabular}

Table 3: Oscillation parameters (first column), values used to construct the pseudo-data sample (second column) and eventual prior (third column). 
A $\chi^{2}$ test has been performed and sensitivity confidence intervals on the reduced parameter space $\sin ^{2}\left(2 \theta_{24}\right)-\Delta m_{41}^{2}$ have been built, by looping over a fine grid of values around the minimum. Results are shown in Figure 4.

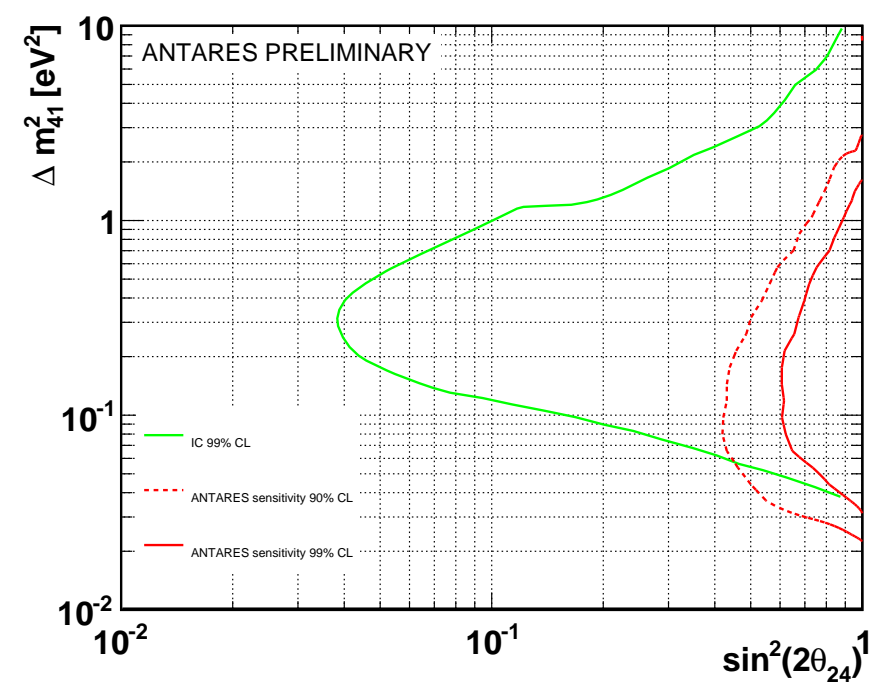

Figure 4: Expected upper limits at 90\% (dashed red) and 99\% (solid red) C.L. for the analysis of our pseudo-data sample. Exclusion regions are on the right side of the lines. For comparison the 99\% C.L. from IceCube [22] is also shown.

It can be seen that the sensitivity regions obtained by the study of our pseudo-data sample are slightly shifted toward lower values of $\Delta m_{41}^{2}$ with respect to the limits presented by the IceCube Collaboration [22]. This can be explained considering the fact that the true energy distribution of our selected events is peaked at lower energy.

\section{Conclusions and Outlook}

A study on the sensitivity of the ANTARES neutrino telescope to the standard atmospheric neutrino oscillation parameters has been performed. A parallel study on the sensitivity to sterile neutrino parameters, in two different energy ranges, has been conducted.

Further improvements of the analysis before the unblinding of real data sets will include the treatment of different sources of systematic effects, in order to study their impact on the final result and a refinement of the quality cuts for selecting events.

\section{References}

[1] ANTARES Collaboration, ANTARES: the first undersea neutrino telescope, Nucl.Instrum.Meth. A656 11-38 (2011).

[2] C. Patrignani et al., Particle Data Group, Chin. Phys. C, 40100001 (2016).

[3] https://github.com/joaoabcoelho/OscProb

[4] ANTARES Collaboration, The Run-by-Run Monte Carlo simulation for the ANTARES experiment, in proceeding of Very Large Volume Neutrino Telescope (VLVnT-2015) 11602002 (2016). 
[5] http:://www.icrr.u-tokyo.ac.jp/ mhonda/nflx2014/index.html

[6] G. Carminati et al., Atmospheric MUons from PArametric formulas: a fast GEnerator for neutrino telescopes (MUPAGE), Comput. Phys. Commun. 179, (915-923), (2008).

[7] Y. Becherini et al., A Parameterisation of single and multiple muons in the deep water or ice, Astropart. Phys. 25 1-13 (2006).

[8] http:://antares.in2p3.fr.

[9] ANTARES Collaboration, A Fast Algorithm for Muon Track Reconstruction and its Application to the ANTARES Neutrino Telescope, Astropart. Phys. 34 (652-662) [astro-ph. IM/1105.4116v1].

[10] E. Visser, Neutrinos from the Milky Way, PhD Thesis, Nikhef (2015).

[11] F. Capozzi et al., Neutrino masses and mixings: Status of known and unknown 3v parameters, for Invited contribution prepared for the Nuclear Physics B Special Issue on Neutrino Oscillations celebrating the Nobel Prize in Physics 2015, hep-ph/1601.07777v1.

[12] A. M. Dziewonski, D. L. Anderson, Preliminary reference Earth model, Physics of the Earth and Planetary Interiors 25 (4) 297-356 (1981).

[13] MINOS Collaboration, Combined Analysis of $v_{\mu}$ Disappearance and $v_{\mu} \rightarrow v_{e}$ Appearance in MINOS Using Accelerator and Atmospheric Neutrinos, Phys. Rev. Lett. 112, 191801 (2014).

[14] NOvA Collaboration, Measurement of the neutrino mixing angle $\theta_{23}$ in NOvA, Phys. Rev. Lett. 118, 151802 (2017) [hep-ex/1701.05891].

[15] T2K Collaboration, Measurements of neutrino oscillation in appearance and disappearance channels by the T2K experiment with $6.6 \times 10^{20}$ protons on target, Phys. Rev. D91 072010 (2015).

[16] A. Terliuk, at XVII International Workshop on Neutrino Telescopes, Venice (2017).

[17] Super-Kamiokande Collaboration, Recent Atmospheric Neutrino Results from Super-Kamiokande in proceeding of $7^{\text {th }}$ International Conference on Interconnection between Particle Physics and Cosmology, 1604 345-352 (2014) [hep-ex/1310. 6677].

[18] MiniBooNE Collaboration, A Combined $v_{\mid} \mu \rightarrow v_{e}$ and $\bar{v}_{\mu} \rightarrow \bar{v}_{e}$ Oscillation Analysis of the MiniBooNE Excesses, hep-ex/1207.4809v2.

[19] IceCube Collaboration, Search for sterile neutrino mixing using three years of IceCube DeepCore data, hep-ex/1702.05160v1.

[20] Super-Kamiokande Collaboration, Limits on sterile neutrino mixing using atmospheric neutrinos in Super-Kamiokande, Phys. Rev. D91 052019 (2015) [hep-ex/1410.2008].

[21] G.D. Barr at al., A three-dimensional calculation of atmospheric neutrinos, Phys. Rev. D70 023006 (2004) [astro-ph/0403630v1].

[22] IceCube Collaboration, Searches for Sterile Neutrinos with the IceCube Detector, Phys. Rev. Lett. 117 071801 (2016) [hep-ex/1605.01990v2]. 